\title{
Lidil
}

Revue de linguistique et de didactique des langues

$55 \mid 2017$

Littéracie et entrée dans l'écrit

Serge Borg, Maria Cheggour, Nadine Desrochers, Laurent Gajo, Vincent Larivière et Monica Vlad (dir.), L'Université en contexte plurilingue dans la dynamique numérique

Éditions des archives contemporaines, 2016, 288 p.

\section{Sílvia Melo-Pfeifer}

\section{OpenEdition}

Journals

Édition électronique

URL : http://journals.openedition.org/lidil/4235

DOI : 10.4000/lidil.4235

ISSN : 1960-6052

Éditeur

UGA Éditions/Université Grenoble Alpes

Édition imprimée

ISBN : 978-2-37747-002-0

ISSN : $1146-6480$

Référence électronique

Sílvia Melo-Pfeifer, « Serge Borg, Maria Cheggour, Nadine Desrochers, Laurent Gajo, Vincent Larivière et Monica Vlad (dir.), L'Université en contexte plurilingue dans la dynamique numérique », Lidil [En ligne], 55 | 2017, mis en ligne le 02 mai 2017, consulté le 25 septembre 2020. URL : http://

journals.openedition.org/lidil/4235; DOI : https://doi.org/10.4000/lidil.4235

Ce document a été généré automatiquement le 25 septembre 2020.

(C) Lidil 


\section{Serge Borg, Maria Cheggour, Nadine Desrochers, Laurent Gajo, Vincent Larivière et Monica Vlad (dir.), L'Université en contexte plurilingue dans la dynamique numérique}

Éditions des archives contemporaines, 2016, 288 p.

Sílvia Melo-Pfeifer

\section{RÉFÉRENCE}

Serge Borg, Maria Cheggour, Nadine Desrochers, Laurent Gajo, Vincent Larivière et Monica Vlad (dir.), L'Université en contexte plurilingue dans la dynamique numérique, Éditions des archives contemporaines, 2016, 288 p.

1 Cette publication est le produit du colloque annuel de l'Agence universitaire de la francophonie (AUF), sous le même titre, tenu à Marrakech en 2015, et organisé autour de trois axes : 1) l'enseignement du français en milieu plurilingue ; 2) le français, langue d'enseignement ; 3) langues et diffusion des savoirs : la communication scientifique. L'ouvrage est structuré autour de ces trois axes, chaque section étant introduite par une réflexion portant sur les aspects transversaux des différentes contributions.

La lecture de ce livre a éveillé en moi la mémoire de P. Neruda et le titre d'un de ses livres les plus connus: Vingt poèmes d'amour et une chanson désespérée. Et cela parce que le livre s'érige en "ode à l'amour » à la langue française et au plurilinguisme "aux temps du choléra ", pour continuer avec les intertextualités. Ainsi, aux potentialités et aux valeurs associées à la langue française en milieu académique, on voit s'ajouter, tout au long des différentes contributions, les obstacles qui freinent - voire menacent son rayonnement et un travail durable dans la production et dans la diffusion des 
savoirs sur et en langue française. Sans être considéré comme un remède, le numérique (dans ses réseaux de formation d'enseignants et d'étudiants, de diffusion et de vulgarisation des savoirs) semble offrir de potentielles voies de résistance à l'incessante monolingualisation des universités (si, comme prérequis, des volontés s'expriment dans ce sens, soit de la part des universités, soit de la part des étudiants et des chercheurs eux-mêmes).

3 Faisant écho à ces constatations, l'ouvrage développe, de façon assez cohérente, trois lignes de force thématiques interdépendantes. La première porte sur l'enseignementapprentissage $\mathrm{du}$ français dans des milieux plurilingues et avec des statuts disciplinaires variés, où l'on peut reconnaitre une progressive perte de «terrain » tant linguistique que symbolique et où l'on prête aux TICE, souvent de façon quelque peu pressée, « toutes les vertus émancipatrices » (F. Barthélémy, p. 20). La deuxième section décrit et analyse l'utilisation du français comme langue d'enseignement à l'université, souvent dans des contextes postcoloniaux (Algérie, Maroc, Liban, Vietnam, Tunisie, Haïti...), en rapport avec la question de la formation initiale et du développement de compétences pédagogiques et linguistiques des enseignants, notamment pour ce qui est de l'usage du numérique comme outil d'innovation. Pour finir, la troisième ligne de force thématique porte sur les défis de la communication scientifique en français, dans un contexte globalisé marqué par une préférence - (in)volontaire et de la part de qui ? - de publication en anglais. Par rapport à ce troisième point, P. Escudé et L. Gajo réfléchissent sur le rôle du français dans la défense d'une "science multipolaire " (p. 166) : tout en reconnaissant l'utilité attachée à la maitrise d'une langue commune, ils affirment qu'il s'agit de s'opposer à une adhésion mécanique à l'anglais qui va « vers l'unanimisme"; il s'agirait de lutter contre ce que ces auteurs nomment la "standardisation", le "capitalisme académique» et la "productification des connaissances » (p. 162), à travers la discussion d'alternatives plurilingues.

Le poids des représentations sociales autour du français et de l'anglais, de leur enseignement, ainsi qu'autour de leurs valeurs économiques et académiques, trouve dans cet ouvrage un fertile terrain de réflexion et de recherche. Finalement, les différentes contributions rendent compte d'expériences de renforcement de la présence du français dans des contextes académiques divers, dans une logique de partage de «bonnes pratiques » montrant, en amont, les tensions entre la globalisation et la contextualisation des politiques linguistiques universitaires, des pratiques pédagogiques et des terrains académiques.

\section{AUTEURS}

\section{SÍLVIA MELO-PFEIFER}

Fakultät für Erziehungswissenschaft, Universität Hamburg 